\title{
ESTIMATIVA DAS NECESSIDADES NUTRICIONAIS DE BANANEIRAS DO SUBGRUPO CAVENDISH CULTIVADAS NO ESTADO DE SÃO PAULO ${ }^{1}$
}

\author{
LUIZANTONIO JUNQUEIRA TEIXEIRA², BERNARDO VAN RAIJ ${ }^{3}$, JOSÉ EMÍLIO BETTIOL NETO ${ }^{4}$
}

RESUMO - A acumulação na planta e a exportação de nutrientes pela colheita dos cachos são alguns dos fatores que determinam a necessidade de adubação para a cultura da bananeira. Visando a estimar as quantidades de nutrientes acumulados e exportados por bananeiras do subgrupo Cavendish, nas condições de cultivo do Estado de São Paulo, foram considerados 293 registros de um banco de dados contendo teores de N, P, K, Ca, Mg, S, B, Cu, Fe, Mn e Zn em frutos e em engaços e massa dos cachos das cultivares Grande Naine e Nanicão. Esses registros provieram de experimentos de adubação realizados no Planalto Paulista e no Vale do Ribeira, em áreas irrigadas e de sequeiro, durante sete ciclos de cultivo, variando fontes, doses e formas de aplicação de fertilizantes. Para produzir $40 \mathrm{t} \mathrm{ha}^{-1}$, em média, o nutriente exportado pelos cachos em maior quantidade foi o $\mathrm{K}\left(182 \mathrm{~kg} \mathrm{ha}^{-1}\right)$ seguido pelo $\mathrm{N}$ (68 $\left.\mathrm{kg} \mathrm{ha}^{-1}\right), \mathrm{Mg}\left(10 \mathrm{~kg} \mathrm{ha}^{-1}\right), \mathrm{P}\left(8 \mathrm{~kg} \mathrm{ha}^{-1}\right), \mathrm{Ca}\left(6 \mathrm{~kg} \mathrm{ha}^{-1}\right), \mathrm{S}\left(3 \mathrm{~kg} \mathrm{ha}^{-1}\right), \mathrm{Mn}\left(191 \mathrm{~g} \mathrm{ha}^{-1}\right), \mathrm{Fe}\left(147 \mathrm{~g} \mathrm{ha}^{-1}\right), \mathrm{B}\left(89 \mathrm{~g} \mathrm{ha}^{-1}\right), \mathrm{Zn}$ $\left(68 \mathrm{~g} \mathrm{ha}^{-1}\right)$ e $\mathrm{Cu}\left(25 \mathrm{~g} \mathrm{ha}^{-1}\right)$. A recomendação de adubação para bananeira para o Estado de São Paulo, aparentemente, subestima a necessidade de $\mathrm{K}$ na implantação da cultura e preconiza doses de $\mathrm{N}$ muito superiores à exportação de $\mathrm{N}$ pelos cachos. Para P, a recomendação está coerente com as necessidades estimadas para a cultura.

Termos para idexação: adubação, fruticultura, nutrição mineral, Musa spp.

\section{ESTIMATE NUTRITION NEEDS OF CAVENDISH BANANA TREES SUBGRUP GROWN IN THE STATE OF SÃO PAULO, BRAZIL}

\begin{abstract}
Fertilizer recommendations for banana take into consideration the accumulation of nutrients in plants and the amount removed in the bunches, among other factors. Nutrient contents accumulated in plants and removed by bunches of Cavendish bananas in the State of São Paulo (Brazil) were assessed from a data bank of nutrient concentrations (N, P, K, Ca, Mg, S, B, Cu, Fe, Mn and $\mathrm{Zn}$ ) in bunches (fruits+peduncle) and bunch weight with 293 samples. Data bank comprises information from plants of Grand Naine and Giant Cavendish cultivars growing in irrigated and non-irrigated areas, with different sources and rates of fertilizers during seven crop cycles. This data came from fertilization experiments accomplished at Planalto Paulista and at the Vale do Ribeira. Potassium was the nutrient most removed by the bunches $\left(182 \mathrm{~kg} \mathrm{ha}^{1}\right)$ followed by N (68 kg ha $\left.{ }^{1}\right), \mathrm{Mg}\left(10 \mathrm{~kg} \mathrm{ha}{ }^{1}\right), \mathrm{P}\left(8 \mathrm{~kg}\right.$ ha $\left.{ }^{1}\right), \mathrm{Ca}$ $\left(6 \mathrm{~kg} \mathrm{ha}^{1}\right), \mathrm{S}\left(3 \mathrm{~kg} \mathrm{ha}^{1}\right), \mathrm{Mn}\left(191 \mathrm{~g} \mathrm{ha}^{1}\right), \mathrm{Fe}\left(147 \mathrm{~g} \mathrm{ha}^{1}\right), \mathrm{B}\left(89 \mathrm{~g} \mathrm{ha}{ }^{1}\right), \mathrm{Zn}\left(68 \mathrm{~g} \mathrm{ha}^{\mathrm{l}}\right)$, and $\mathrm{Cu}\left(25 \mathrm{~g} \mathrm{ha}^{1}\right)$ for an average bunch yield of $40 \mathrm{t} \mathrm{ha}^{-1}$. Fertilizer recommendations for banana in the State of São Paulo underestimate K requirements for plant establishing. The recommended rates of $\mathrm{N}$ are higher than $\mathrm{N}$ removal by bunches. The recommendation of $\mathrm{P}$ fertilizer is in accordance with plant requirements.
\end{abstract}

Index terms: fertilizer recommendation, fruit crops, mineral nutrition, Musa spp.

\section{INTRODUÇÃO}

A bananeira é uma planta de crescimento rápido e que necessita de concentrações elevadas de nutrientes disponíveis no solo para seu desenvolvimento e produção normais. Esses podem ser fornecidos, em parte, pelo solo e pela ciclagem no sistema solo-planta, porém, para obtenção de produções economicamente viáveis, essas fontes normalmente não são suficientes, sendo imprescindível a aplicação de fertilizantes em quantidades e proporções adequadas para suprir os nutrientes exigidos pela cultura (Lahav \& Turner, 1989; Soto, 1992).

Segundo Turner \& Barkus (1982), conhecer a composição química dos cachos é importante para estimar a reposição dos nutrientes exportados pela cultura. Para cultivos comerciais, com rendimentos de $50 \mathrm{t} \mathrm{ha}^{-1}$ ano $^{-1}$, em solos minerais, e empregandose variedades do subgrupo Cavendish, Lahav (1995) apresentou os seguintes valores médios para exportação de nutrientes pelos cachos: $189 \mathrm{~kg} \mathrm{ha}^{1}$ ano $^{1}$ de N, 778 de K, 29 de P, $101 \mathrm{de} \mathrm{Ca}, 49$ de $\mathrm{Mg}, 23$ de S, 0,7 de B, 0,2 de Cu, 0,9 de Fe, 0,5 de Mn e 0,5 de Zn. Em cultivos de alto rendimento $\left(70 \mathrm{tha}^{-1} \mathrm{ano}^{-1}\right)$ e para as condições da América Central, López \& Espinosa (1995) estimaram que a quantidade de nutrientes exportada com os cachos foi superior a $125 \mathrm{~kg} \mathrm{ha}^{-1}$ ano $^{-1} \mathrm{de} \mathrm{N}, 400$ de K e 15 de P. Estes autores destacaram que a manutenção de rendimentos elevados ao longo do tempo depende da reposição dos nutrientes exportados por meio de adubações.

No Brasil, Borges \& Oliveira (2000), trabalhando com sete cultivares de bananeira na região Nordeste, estimaram que a exportação foi de $1,9 \mathrm{~kg}$ de $\mathrm{N}$ por tonelada de cachos; de $0,22 \mathrm{~kg}$ $\mathrm{t}^{-1}$ de P e de 5,6 $\mathrm{kg} \mathrm{t}^{-1}$ de K. Segundo Malburg \& Lichtemberg (1986), a exportação de nutrientes pela cultura, especialmente N, $\mathrm{P}$ e K, quando expressa por tonelada de cachos, apresenta pouca variação, ainda que estejam envolvidas diferentes condições

\footnotetext{
1'(Trabalho 187-07). Recebido em: 01-08-2007. Aceito para publicação em: 18-12-2007. Apoio: FAPESP (Processos:96/4209-4, 01/09976-3). ${ }_{2}^{2}$ Pesquisador do Centro de Solos e Recursos Ambientais, Instituto Agronômico. Caixa Postal 28, Campinas-SP, 13012-970. teixeira@iac.sp.gov.br. ${ }^{3}$ Pesquisador Voluntário do Centro de Solos e Recursos Ambientais, Instituto Agronômico.Caixa Postal 28, Campinas-SP, 13012-970. bvanraij@iac.sp.gov.br. ${ }^{4}$ Pesquisador do Centro de Fruticultura, Instituto Agronômico. Caixa Postal 28, Campinas-SP, CEP 13012-970. bettiolneto@iac.sp.gov.br.
} 
ambientais, cultivares ou épocas. Esses autores determinaram para 'Nanicão', em amostragens realizadas em várias épocas, que as quantidades de N, P e K acumuladas nos cachos foram de 1,$9 ; 0,21$ e $3,85 \mathrm{~kg}$ por tonelada, respectivamente.

A necessidade de adubação da cultura é função da exportação de nutrientes pelos cachos, entre outros fatores. A quantidade de nutrientes contidos nos cachos tem sido objeto de estimativa em vários trabalhos, entretanto há grande variação entre os valores determinados pelos diversos autores. Fatores como variedade, manejo da cultura, condições edafoclimáticas e métodos de amostragem empregados em cada trabalho têm efeito sobre os resultados obtidos. Ainda que aproximados e variando em função das condições de cultivo, tais valores chamam a atenção pela sua magnitude, especialmente em relação ao potássio e, em menor grau, ao nitrogênio. Lahav \& Turner (1989) compilaram várias recomendações de adubação para bananeira utilizadas em diversas regiões produtoras do mundo, destacandose as elevadas doses de $\mathrm{N} \mathrm{e} \mathrm{K}$, as quais, segundo os autores, devem-se, em parte, à quantidade destes nutrientes que é exportada pelos cachos.

Para o Estado de São Paulo, a recomendação de adubação inclui a produtividade esperada (PE) como um dos critérios para determinação das doses dos fertilizantes. Quaggio \& Raij (1997) justificaram a importância de considerar a PE, pois culturas mais produtivas requerem maior quantidade de nutrientes e, com maiores produções, há maior renda, o que permite a aquisição de mais fertilizante. De certa forma, a necessidade de incluir diferentes aspectos que influem na necessidade de fertilizantes, como cultivares e manejo do bananal, foi contornada com a introdução do conceito de PE. Atualmente, em São Paulo, considera-se apenas a PE na recomendação de $\mathrm{N}$ para bananeira; na determinação das doses de $\mathrm{P}$ e $\mathrm{K}$, além da produtividade, a disponibilidade destes nutrientes no solo também influi na quantidade de adubo a ser aplicada, conforme Boletim Técnico do Instituto Agronômico (BT 100/IAC), apresentado por Teixeira et al. (1997), de onde foi extraída a Tabela 1.

O objetivo deste trabalho foi estimar a quantidade de nutrientes acumulados nas plantas e exportados com a colheita dos cachos de bananeiras do subgrupo Cavendish nas condições de cultivo do Estado de São Paulo e comparar as necessidades nutricionais estimadas com a recomendação de adubação.

\section{MATERIAL E MÉTODOS}

As estimativas de exportação de nutrientes pela colheita dos cachos (frutos+engaço) foram obtidas a partir de um banco de dados com 293 registros, referentes às cultivares Grande Naine e Nanicão, ambas pertencentes ao Grupo AAA e subgrupo Cavendish. Estes registros foram coletados em experimentos de adubação realizados no Planalto Paulista (Latossolo Vermelho eutroférrico; Argissolo Vermelho-Amarelo) e no Vale do Ribeira (Latossolo Amarelo distrófico), em áreas irrigadas e de sequeiro, com variação nas fontes (orgânica e mineral) e doses de fertilizantes, durante sete ciclos de cultivo. Todas as áreas experimentais foram plantadas no espaçamento de $2,0 \times 2,5 \mathrm{~m}$ (2.000 famílias por hectare) e manejadas de acordo com as recomendações técnicas para a cultura.

A exportação de nutrientes pelos cachos foi estimada analisando-se os teores de N, P, K, Ca, Mg, S, B, Cu, Fe, Mn e Zn de acordo com Bataglia et al. (1983) e a produção de matéria seca, dos frutos e engaço, em separado. Para tanto, coletaram-se seis frutos por cacho (terço superior, meio e terço inferior), formando duas amostras de frutos compostas, sendo uma para análise química, e outra, para determinação de matéria seca. Foram coletadas quatro seções transversais do engaço com $\pm 3 \mathrm{~cm}$ de espessura (duas na metade superior e duas na inferior), para formar duas amostras compostas, nas quais se fez análise química e quantificou-se a umidade. Os resultados de N, K, P, Ca, Mg e S foram expressos em $\mathrm{kg} \mathrm{ha}^{-1} \mathrm{e}$ em $\mathrm{kg}$ por tonelada de cachos frescos; para os micronutrientes, em $\mathrm{g} \mathrm{ha}^{-1} \mathrm{e}$ em $\mathrm{g}$ por tonelada de cachos frescos. As médias obtidas em diferentes regimes hídricos (irrigado e sequeiro) foram comparadas empregando-se o teste $\mathrm{t}$ de Student $(\alpha=0,05)$.

A acumulação de nutrientes (N, P e K) nas plantas foi estimada de modo indireto, admitindo-se que há uma proporção entre as quantidades de nutrientes exportadas pelos cachos e a acumulação na biomassa das plantas (planta-mãe e rebento seguidor) relativamente estável para cada variedade ou subgrupo. Empregou-se a relação apresentada por Marchal \& Mallessard (1979) para 'Grande Naine' (AAA, subgrupo Cavendish), a qual foi estimada a partir de teores e produtividades da mesma ordem de grandeza dos que foram obtidos no presente trabalho. Segundo esses autores, a proporção entre a quantidade acumulada no cacho e a imobilizada na biomassa da planta foi para N 1:2,7, para P foi de 1:2,3 e K, 1:3,1.

As estimativas de acumulação de nutrientes nas plantas e de exportação com a colheita dos cachos foram comparadas com as doses de $\mathrm{N}, \mathrm{P}$ e $\mathrm{K}$ recomendadas para bananais cultivados no Estado de São Paulo, apresentadas no BT 100/IAC por Teixeira et al. (1997) (Tabela 1). Comparou-se a quantidade de nutrientes acumulada nas plantas com as doses recomendadas para a adubação inicial aplicada na formação do bananal. A exportação de nutrientes pelos cachos foi cotejada com a recomendação de adubação de manutenção aplicada anualmente.

\section{RESULTADOS E DISCUSSÃO}

Na Tabela 2, encontram-se as médias, amplitudes e desvios-padrão da quantidade de $\mathrm{N}, \mathrm{K}, \mathrm{P}, \mathrm{Ca}, \mathrm{Mg}, \mathrm{S}, \mathrm{B}, \mathrm{Cu}, \mathrm{Fe}$, $\mathrm{Mn}$ e $\mathrm{Zn}$ exportada pelos cachos e da produtividade das bananeiras obtidas a partir do banco de dados. A escala de exportação de nutrientes, em ordem decrescente, foi: $\mathrm{K}>\mathrm{N}>\mathrm{Mg}$ $>\mathrm{P}>\mathrm{Ca}>\mathrm{S}>\mathrm{Mn}>\mathrm{Fe}>\mathrm{B}>\mathrm{Zn}>\mathrm{Cu}$. Esses valores são significativamente menores do que os compilados por Lahav (1995) e por López \& Espinosa (1995) para remoção de nutrientes pelos cachos, os quais foram gerados a partir de experimentos ou áreas de produção localizados na América Central. Nessas áreas, além de altas produtividades, são comuns solos com elevada disponibilidade de potássio (originários de cinzas vulcânicas ou deposição aluvional) e ricos em matéria orgânica, nas quais, provavelmente, esteja ocorrendo consumo de luxo, especialmente de potássio. 
Os valores médios de exportação de nutrientes por tonelada de cachos (Tabela 2) são da mesma ordem de grandeza que os apresentados por Malburg \& Lichtemberg (1986) e por Borges \& Oliveira (2000). De acordo com Malburg \& Lichtemberg (1986), as diferenças devidas às condições de cultivo tendem a diminuir quando a remoção é expressa por tonelada de cachos. Turner \& Barkus (1982) observaram em experimento de campo com a bananeira 'Williams' que a concentração de K nos cachos variou pouco em função do teor de K no solo e que a exportação desse elemento seria, portanto, função direta do rendimento.

Observa-se na Tabela 2 que, mesmo expressando a exportação de nutrientes por tonelada de frutos, a amplitude dos valores ainda foi relativamente grande. Essa variação pode ser creditada, em parte, à diversidade das condições de cultivo dos bananais de onde provieram os registros do banco de dados. Visando a verificar se havia variação na exportação de nutrientes por tonelada em função da massa dos cachos, fez-se o estudo de correlação apresentado na Tabela 3. A exportação de $\mathrm{N}, \mathrm{Mg}, \mathrm{Cu}$, $\mathrm{Fe}$ e $\mathrm{Mn}$, expressa por tonelada produzida, variou inversamente com a massa dos cachos, enquanto a exportação de K, também em $\mathrm{kg} \mathrm{t}^{1}$, foi diretamente proporcional. A diminuição dos teores de nutrientes com o aumento da produção indica efeito de diluição, mas o aumento na concentração de $\mathrm{K}$ nos cachos maiores pode estar associado a consumo de luxo. De qualquer forma, esta observação ratifica a necessidade de monitoramento da fertilidade do solo, especialmente dos teores de K disponível, visando a manter o adequado suprimento deste nutriente às plantas ao longo do tempo.

Para testar se o regime hídrico teve alguma influência na exportação de nutrientes expressa por tonelada de cachos, fezse a comparação das médias apresentada na Tabela 4. A ordem de grandeza dos valores, tanto das áreas sob irrigação como nas de sequeiro, é semelhante. Entretanto, observaram-se diferenças estatisticamente significativas para as quantidades de $\mathrm{K}, \mathrm{P}, \mathrm{Ca}$, $\mathrm{Mg}, \mathrm{Cu}$ e $\mathrm{Zn}$.

$\mathrm{Na}$ Tabela 5, é apresentada a estimativa de exportação de nutrientes pelos cachos num bananal com 2.000 famílias por hectare em função da produtividade obtida a partir do banco de dados em estudo. Na Tabela 6, têm-se as estimativas de acumulação de nutrientes na biomassa das plantas geradas a partir das proporções entre nutrientes exportados e acumulados apresentadas por Marchal \& Mallessard (1979). Os resultados contidos nestas tabelas foram empregados no cálculo dos balanços apresentados nas Tabelas 7 e 8 .
O balanço entre a quantidade de nutrientes acumulada na biomassa e as doses de fertilizantes recomendadas no BT 100/IAC (Teixeira et al., 1997) para aplicação na implantação de um bananal é apresentado na Tabela 7. Observa-se que, para nitrogênio e fósforo, o balanço é positivo. Aplica-se mais $\mathrm{N}$ do que a necessidade das plantas, pois usualmente a eficiência da adubação nitrogenada é baixa. Para fósforo, a recomendação é coerente com a acumulação nas plantas. Nas classes de teores mais baixos de $\mathrm{P}$ disponível no solo, o balanço é mais positivo, o que se justifica pelo interesse em aumentar a disponibilidade de $\mathrm{P}$ com as sucessivas adubações. Já para as classes de teores mais altos, a recomendação fica mais próxima da quantidade acumulada nas plantas. Em relação ao potássio, elemento-chave da nutrição das bananeiras, a recomendação do BT 100/IAC (Tabela 1) é insuficiente para suprir sua acumulação na biomassa das plantas no, estabelecimento do bananal, pois o balanço é negativo para quase todas as classes de disponibilidade no solo e de produtividade. Essa observação pode implicar que a produtividade das plantas, na primeira safra seja limitada pelo suprimento inadequado de $\mathrm{K}$, especialmente em solos com baixa disponibilidade deste nutriente.

Comparando-se a recomendação de adubação de manutenção do bananal, que visa a repor os nutrientes exportados pelos cachos em cada safra com a quantidade de nutrientes exportados com a colheita (Tabela 5), observa-se que o balanço é positivo para todas as situações (Tabela 8). As doses recomendadas de $\mathrm{N}, \mathrm{P}$ e $\mathrm{K}$ são superiores à exportação, destacando-se doses de $\mathrm{N}$ até cinco vezes maiores do que a quantidade exportada. Mesmo com a baixa eficiência da adubação nitrogenada, pode-se estar incorrendo em excessos, especialmente se forem adotadas práticas que aumentem a eficiência do $\mathrm{N}$ aplicado, como a fertirrigação. A aplicação de doses de $\mathrm{N}$ acima das exigidas pela planta representa não só desperdício e risco de contaminação ambiental, como também pode causar a produção de cachos menores e com problemas de enchimento dos frutos, apesar da aparência sadia das plantas, como descrito por Robinson (1996). A queda de frutos das pencas já amadurecidas (finger drop) tem sido associada à nutrição desbalanceada de nitrogênio. Esse problema de pós-colheita é comum em áreas tropicais, na estação úmida e com baixo suprimento de potássio. O desequilíbrio nutricional de $\mathrm{N}$ e K (Nfoliar alto e K-foliar baixo) pode também acelerar a senescência das folhas entre a época da emissão da inflorescência e a colheita dos cachos (Teixeira et al., 2001).

TABELA 1-Recomendação de adubação para bananeira (BT 100/IAC) com base na produtividade esperada e nos teores de $\mathrm{P}$ e K disponíveis no solo apresentada por Teixeira et al. (1997).

\begin{tabular}{|c|c|c|c|c|c|c|c|c|c|c|c|c|}
\hline \multirow{2}{*}{$\begin{array}{c}\text { Produtividade } \\
\text { esperada }\end{array}$} & \multirow{2}{*}{$\mathbf{N}$} & \multicolumn{4}{|c|}{$P$ resina, $\mathrm{mg} / \mathrm{dm}^{3}$} & \multicolumn{4}{|c|}{$\mathrm{K}^{+}$trocável, $\mathbf{m m o l}_{\mathrm{c}} / \mathbf{d m}^{3}$} & \multirow{2}{*}{$\mathbf{B}^{(\mathbf{1})}$} & \multirow{2}{*}{$\mathbf{Z n}^{(2)}$} & \multirow{2}{*}{$\mathbf{S}^{(\mathbf{3})}$} \\
\hline & & $0-5$ & 6-12 & 13-30 & $>\mathbf{3 0}$ & 0-0,7 & 0,8-1,5 & $1,6-3,0$ & $>\mathbf{3 , 0}$ & & & \\
\hline t/ha & $\mathrm{kg} / \mathrm{ha}$ & \multicolumn{4}{|c|}{--------- $\mathrm{P}_{2} \mathrm{O}_{5}, \mathrm{~kg} / \mathrm{ha}$--------- } & \multicolumn{4}{|c|}{-------- $\mathrm{K}_{2} \mathrm{O}, \mathrm{kg} / \mathrm{ha}$} & \multicolumn{3}{|c|}{---------- kg/ha ---------- } \\
\hline$<20$ & 120 & 80 & 60 & 40 & 20 & 330 & 230 & 130 & 90 & 3,4 & 10 & 30 \\
\hline $20-30$ & 190 & 100 & 80 & 50 & 30 & 410 & 310 & 210 & 150 & 3,4 & 10 & 30 \\
\hline $30-40$ & 270 & 140 & 110 & 70 & 40 & 490 & 390 & 290 & 210 & 3,4 & 10 & 30 \\
\hline $40-50$ & 350 & 180 & 140 & 90 & 50 & 570 & 470 & 370 & 270 & 3,4 & 10 & 30 \\
\hline $50-60$ & 430 & 220 & 170 & 110 & 60 & 650 & 550 & 450 & 330 & 3,4 & 10 & 30 \\
\hline$>60$ & 500 & 260 & 200 & 130 & 70 & 730 & 630 & 530 & 390 & 3,4 & 10 & 30 \\
\hline
\end{tabular}

Fracionamento, fontes e modo de aplicação dos fertilizantes estão descritos no Boletim Técnico 100 do IAC (Teixeira et al., 1997).

(1) $10 \mathrm{~g}$ de ac. bórico por planta no momento do desbaste, ${ }^{(2)} 25 \mathrm{~g}$ de sulfato de zinco por planta no momento do desbaste; os micronutrientes só devem ser aplicados quando for diagnosticada deficiência. ${ }^{(3)}$ Utilizar fontes de $\mathrm{N}$ ou P capazes de fornecer, anualmente, $30 \mathrm{~kg} / \mathrm{ha}$ de $\mathrm{S}$.

Rev. Bras. Frutic., Jaboticabal - SP, v. 30, n. 2, p. 540-545, Junho 2008 
TABELA 2- Estatísticas do banco de dados com 293 registros de exportação de N, K, P, Ca, Mg, S, B, Cu, Fe, Mn e Zn pelos cachos e produtividade de bananeira do subgrupo Cavendish com 2.000 famílias por hectare.

\begin{tabular}{|c|c|c|c|c|c|c|c|c|}
\hline Variável & Média & Mínimo & Máximo & $\begin{array}{l}\text { Desvio- } \\
\text { padrão }\end{array}$ & Média & Mínimo & Máximo & $\begin{array}{l}\text { Desvio- } \\
\text { padrão }\end{array}$ \\
\hline & \multicolumn{4}{|c|}{------------------------ kg ha ${ }^{-1}$-------------------------- } & \multicolumn{4}{|c|}{ - } \\
\hline $\mathbf{N}$ & 68 & 22 & 137 & 21,4 & 1,75 & 0,87 & 2,59 & 0,317 \\
\hline $\mathbf{K}$ & 182 & 31 & 375 & 65,5 & 4,50 & 3,01 & 6,89 & 0,569 \\
\hline $\mathbf{P}$ & 8 & 2 & 16 & 2,9 & 0,20 & 0,12 & 0,31 & 0,035 \\
\hline $\mathbf{C a}$ & 6 & 1 & 21 & 3,2 & 0,14 & 0,03 & 0,37 & 0,055 \\
\hline $\mathbf{M g}$ & 10 & 3 & 23 & 3,2 & 0,24 & 0,15 & 0,35 & 0,032 \\
\hline \multirow[t]{2}{*}{$\mathbf{S}$} & 3 & 1 & 6 & 1,1 & 0,09 & 0,05 & 0,12 & 0,013 \\
\hline & \multicolumn{4}{|c|}{$\mathrm{g} \mathrm{ha}^{-1}$} & & & & 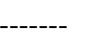 \\
\hline B & 89 & 25 & 260 & 40,5 & 2,23 & 0,63 & 4,06 & 0,664 \\
\hline $\mathbf{C u}$ & 25 & 4 & 69 & 9,6 & 0,67 & 0,23 & 2,19 & 0,304 \\
\hline $\mathbf{F e}$ & 147 & 20 & 890 & 123,8 & 3,82 & 0,53 & 25,05 & 2,889 \\
\hline Mn & 191 & 32 & 783 & 143,1 & 5,03 & 0,56 & 21,26 & 3,616 \\
\hline $\mathbf{Z n}$ & 68 & 17 & 202 & 33,1 & 1,68 & 0,80 & 5,54 & 0,624 \\
\hline Produtividade & 40,1 & 9,8 & 73,6 & 13,0 & & & & \\
\hline
\end{tabular}

TABELA 3- Coeficientes de correlação de Pearson (R) e valores $p$ associados $(p)$ à correlação entre as quantidades de nutrientes exportados por tonelada de cachos $\left(\mathbf{N}_{\text {exp }} \ldots \mathbf{Z n}_{\text {exp }}\right)$ e a massa dos cachos de bananeira do subgrupo Cavendish.

\begin{tabular}{|c|c|c|c|c|c|c|c|c|c|c|}
\hline $\mathbf{N}_{\text {exp }}$ & $K_{\text {exp }}$ & $\mathbf{P}_{\text {exp }}$ & $\mathbf{C a} \mathbf{a}_{\exp }$ & $\mathbf{M g}_{\text {exp }}$ & $\mathbf{S}_{\text {exp }}$ & $\mathbf{B}_{\text {exp }}$ & $\mathbf{C} \mathbf{u}_{\text {exp }}$ & $\mathbf{F e}_{\text {exp }}$ & $\mathbf{M n} \mathbf{n}_{\text {exp }}$ & $\mathbf{Z} \mathbf{n}_{\text {exp }}$ \\
\hline & ------ & & & 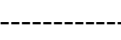 & --- R --. & - & ----------. & ---------. & ------ & \\
\hline$-0,4378$ & 0,1770 & 0,0043 & 0,0446 & $-0,2055$ & $-0,1191$ & $-0,0005$ & $-\mathbf{0 , 5 0 0 8}$ & $-\mathbf{0 , 1 5 3 7}$ & $-0,2362$ & 0,0304 \\
\hline$<\mathbf{0 , 0 0 1}$ & 0,002 & 0,941 & 0,447 & $<0,001$ & 0,184 & 0,993 & $<0,001$ & 0,008 & $<0,001$ & 0,604 \\
\hline
\end{tabular}

Valores em negrito são significativos a $5 \%$ de probabilidade $(p<0,05)$.

TABELA 4- Valores médios das quantidades de nutrientes exportados por tonelada de cachos em bananeira do subgrupo Cavendish em sequeiro e sob irrigação.

\begin{tabular}{|c|c|c|c|c|c|}
\hline \multirow{2}{*}{ Exportação } & \multicolumn{2}{|c|}{ Produção em sequeiro } & \multicolumn{2}{|c|}{ Produção sob irrigação } & \multirow{2}{*}{$\begin{array}{c}\text { Teste t para médias } \\
\text { Valor } p^{(2)}\end{array}$} \\
\hline & Média & $\mathbf{D P}^{(1)}$ & Média & DP & \\
\hline$N\left(\operatorname{kg~t}^{-1}\right)$ & 1,780 & 0,330 & 1,715 & 0,315 & 0,095 \\
\hline$K\left(\mathrm{~kg} \mathrm{t}^{-1}\right)$ & 4,427 & 0,610 & 4,612 & 0,484 & 0,006 \\
\hline$P\left(\mathrm{~kg} \mathrm{t}^{-1}\right)$ & 0,191 & 0,034 & 0,212 & 0,032 & $<0,001$ \\
\hline $\mathrm{Ca}\left(\mathrm{kg} \mathrm{t}^{-1}\right)$ & 0,153 & 0,058 & 0,118 & 0,043 & $<0,001$ \\
\hline$M g\left(\operatorname{kg~t}^{-1}\right)$ & $\mathbf{0 , 2 3 9}$ & 0,033 & 0,251 & 0,028 & 0,001 \\
\hline$S\left(\operatorname{kg~t}^{-1}\right)$ & 0,091 & 0,012 & 0,087 & 0,014 & 0,083 \\
\hline $\mathbf{B}\left(\mathrm{g} \mathrm{t}^{-1}\right)$ & 2,24 & 0,662 & 2,21 & 0,667 & 0,672 \\
\hline $\mathrm{Cu}\left(\mathrm{g} \mathrm{t}^{-1}\right)$ & 0,71 & 0,330 & 0,61 & 0,251 & 0,005 \\
\hline $\mathrm{Fe}\left(\mathrm{g} \mathrm{t}^{-1}\right)$ & 4,05 & 3,128 & 3,52 & 2,487 & 0,121 \\
\hline $\operatorname{Mn}\left(g^{-1}\right)$ & 4,75 & 4,153 & 5,43 & 2,631 & 0,115 \\
\hline $\operatorname{Zn}(\mathrm{g}$ t-1) & $\mathbf{1 , 8 0}$ & 0,689 & 1,52 & 0,472 & $\mathbf{0 , 0 0 1}$ \\
\hline
\end{tabular}

${ }^{(1)}$ desvio-padrão; ${ }^{(2)}$ probabilidade do erro tipo I associada ao teste t ou nível de significância do teste; médias de um mesmo nutriente em negrito diferem estatisticamente, a $5 \%$ de probabilidade $(p<0,05)$.

TABELA 5 - Exportação de nutrientes pelos cachos em bananal com 2.000 famílias por hectare em função da produtividade.

\begin{tabular}{|c|c|c|c|c|c|c|c|c|c|c|c|}
\hline Produtividade & $\mathbf{N}$ & $\mathbf{K}_{2} \mathbf{O}$ & $\mathbf{P}_{2} \mathbf{O}_{5}$ & $\mathbf{C a}$ & Mg & $\mathbf{S}$ & B & $\mathbf{C u}$ & $\mathbf{F e}$ & Mn & $\mathbf{Z n}$ \\
\hline t/ha & \multicolumn{6}{|c|}{ 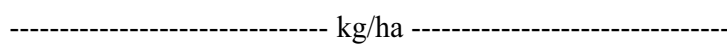 } & \multicolumn{5}{|c|}{ 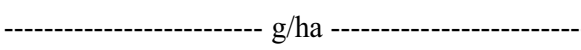 } \\
\hline 15 & 31 & 70 & 6 & 2 & 4 & 1 & 34 & 14 & 74 & 86 & 25 \\
\hline 25 & 49 & 135 & 11 & 3 & 6 & 2 & 55 & 21 & 113 & 137 & 37 \\
\hline 35 & 61 & 192 & 17 & 5 & 9 & 3 & 79 & 26 & 110 & 246 & 61 \\
\hline 45 & 77 & 244 & 20 & 6 & 11 & 4 & 104 & 26 & 189 & 205 & 86 \\
\hline 55 & 86 & 304 & 25 & 8 & 13 & 5 & 116 & 27 & 207 & 187 & 86 \\
\hline 65 & 101 & 358 & 30 & 9 & 15 & 5 & 142 & 31 & 201 & 213 & 100 \\
\hline
\end{tabular}


TABELA 6 - Estimativa de acumulação de nutrientes na biomassa de bananal com 2.000 famílias por hectare em função da produtividade. Valores gerados a partir das proporções entre nutrientes exportados e acumulados apresentadas por Marchal \& Mallessard (1979).

\begin{tabular}{cccc}
\hline Produtividade & $\mathbf{N}$ & $\mathbf{K}_{\mathbf{2}} \mathbf{O}$ & $\mathbf{P}_{\mathbf{2}} \mathbf{O}_{5}$ \\
\hline & ----------- & $\mathbf{k g} / \mathbf{h a}$ & ---------- \\
$\mathbf{1 5}$ & 84 & 218 & 15 \\
$\mathbf{3 5}$ & 132 & $42 \mathrm{O}$ & 26 \\
$\mathbf{4 5}$ & 164 & 595 & 39 \\
$\mathbf{5 5}$ & 208 & 757 & 46 \\
$\mathbf{6 5}$ & 231 & 942 & 57 \\
\hline
\end{tabular}

TABELA 7- Balanço entre as doses de fertilizantes recomendadas no BT 100/IAC (Teixeira et al., 1997) para aplicação na implantação de bananal com 2.000 famílias por hectare e a quantidade de nutrientes acumulados na biomassa das bananeiras.

\begin{tabular}{|c|c|c|c|c|c|c|c|c|c|}
\hline \multirow{2}{*}{ Produtividade } & \multirow{2}{*}{$\mathbf{N}$} & \multicolumn{4}{|c|}{ P resina, $\mathrm{mg} / \mathrm{dm}^{3}$} & \multicolumn{4}{|c|}{$\mathrm{K}^{+}$trocável, $\mathbf{m m o l}_{\mathrm{c}} / \mathrm{dm}^{3}$} \\
\hline & & $0-5$ & 6-12 & 13-30 & $>\mathbf{3 0}$ & $0-0,7$ & $\mathbf{0 , 8 - 1 , 5}$ & $1,6-3,0$ & $>\mathbf{3 , 0}$ \\
\hline t/ha & $\mathrm{kg} / \mathrm{ha}$ & \multicolumn{4}{|c|}{--------------- $\mathrm{P}_{2} \mathrm{O}_{5}, \mathrm{~kg} / \mathrm{ha}$--------------- } & \multicolumn{4}{|c|}{ 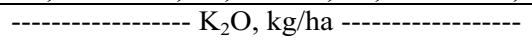 } \\
\hline 15 & 36 & 65 & 45 & 25 & 5 & 112 & 112 & -88 & -128 \\
\hline 25 & 58 & 74 & 54 & 24 & 4 & -10 & -110 & -210 & -270 \\
\hline 35 & 106 & 101 & 71 & 31 & 1 & -105 & -205 & -305 & -385 \\
\hline 45 & 142 & 134 & 94 & 44 & 4 & -187 & -287 & -387 & -487 \\
\hline 55 & 199 & 163 & 113 & 53 & 3 & -292 & -392 & -492 & -612 \\
\hline 65 & 227 & 191 & 131 & 61 & 1 & -378 & -478 & -578 & -718 \\
\hline
\end{tabular}

TABELA 8- Balanço entre as doses de fertilizantes recomendadas no BT 100/IAC (Teixeira et al., 1997) para aplicação anual e a quantidade de nutrientes exportados pelos cachos.

\begin{tabular}{|c|c|c|c|c|c|c|c|c|c|c|c|c|}
\hline \multirow{2}{*}{ Produtividade } & \multirow{2}{*}{$\mathbf{N}$} & \multicolumn{4}{|c|}{$P$ resina, $\mathrm{mg} / \mathrm{dm}^{3}$} & \multicolumn{4}{|c|}{$\mathrm{K}^{+}$trocável, $\mathrm{mmol}_{\mathrm{c}} / \mathrm{dm}^{3}$} & \multirow[b]{2}{*}{ B } & \multirow{2}{*}{ Zn } & \multirow{2}{*}{$\mathbf{S}$} \\
\hline & & $0-5$ & 6-12 & 13-30 & $>\mathbf{3 0}$ & 0-0,7 & $0,8-1,5$ & $1,6-3,0$ & $>\mathbf{3 , 0}$ & & & \\
\hline t/ha & $\mathrm{kg} / \mathrm{ha}$ & \multicolumn{4}{|c|}{--------- $\mathrm{P}_{2} \mathrm{O}_{5}, \mathrm{~kg} / \mathrm{ha}$--------- } & \multicolumn{4}{|c|}{------------- $\mathrm{K}_{2} \mathrm{O}, \mathrm{kg} / \mathrm{ha}$---------- } & \multicolumn{3}{|c|}{----------- kg/ha ----------- } \\
\hline 25 & 141 & 89 & 69 & 39 & 19 & 275 & 175 & 75 & 15 & 3,35 & 9,96 & 27,8 \\
\hline 35 & 209 & 123 & 93 & 53 & 23 & 298 & 198 & 98 & 18 & 3,32 & 9,94 & 26,8 \\
\hline 45 & 273 & 160 & 120 & 70 & 30 & 326 & 226 & 126 & 26 & 3,30 & 9,91 & 26,0 \\
\hline
\end{tabular}

\section{CONCLUSÕES}

1-Para produzir $40 \mathrm{tha}^{-1}$, em média, o nutriente exportado pelos cachos em maior quantidade foi o $\mathrm{K}\left(182 \mathrm{~kg} \mathrm{ha}^{-1}\right)$, seguido pelo $\mathrm{N}\left(68 \mathrm{~kg} \mathrm{ha}^{-1}\right), \operatorname{Mg}\left(10 \mathrm{~kg} \mathrm{ha}^{-1}\right), \mathrm{P}\left(8 \mathrm{~kg} \mathrm{ha}^{-1}\right), \mathrm{Ca}\left(6 \mathrm{~kg} \mathrm{ha}^{-1}\right)$, $\mathrm{S}\left(3 \mathrm{~kg} \mathrm{ha}^{-1}\right), \mathrm{Mn}\left(191 \mathrm{~g} \mathrm{ha}^{-1}\right), \mathrm{Fe}\left(147 \mathrm{~g} \mathrm{ha}^{-1}\right), \mathrm{B}\left(89 \mathrm{~g} \mathrm{ha}^{-1}\right)$, $\mathrm{Zn}\left(68 \mathrm{~g} \mathrm{ha}^{-1}\right)$ e $\mathrm{Cu}\left(25 \mathrm{~g} \mathrm{ha}^{-1}\right)$.

2-A recomendação de adubação para bananeira, para o Estado de São Paulo, aparentemente subestima a necessidade de K na implantação da cultura e preconiza doses anuais de $\mathrm{N}$ muito superiores à exportação de $\mathrm{N}$ pelos cachos. Para $\mathrm{P}$, a recomendação está coerente com as necessidades estimadas para a cultura.

\section{REFERÊNCIAS}

BATAGLIA, O.C.; FURLANI, A.M.C.; TEIXEIRA, J.P.F.; FURLANI, P.R.; GALLO, J.R. Métodos de análise química de plantas. Campinas: IAC, 1983.48p. (Boletim Técnico, 78)

BORGES, A.L.; OLIVEIRA, A.M.G. Nutrição, adubação e calagem. In: CORDEIRO, Z.J.M. (Ed.). Banana: produção: aspectos técnicos. Brasília: Embrapa, 2000. p. 47-59.

LAHAV, E.; TURNER, D.W. Banana. 2.ed. Berna: IPI, 62p. 1989. (Bulletin, 7)

LAHAV, E. Banana nutrition. In: GOWEN, S. (Ed.) Bananas and plantains. London: Chapman \& Hall, 1995. p.258-316. 
LÓPEZ, A.; ESPINOSA, J. Manual de nutricion y fertilizacion del banano. Quito: Corbana/Inpofos, 1995. 82p.

MALBURG, J.L.; LICHTEMBERG, L.A. Composição mineral de frutos de banana-variação estacional do teor de macroelementos na cv. Nanicão em Santa Catarina. In: CONGRESSO BRASILEIRO DE FRUTICULTURA, 8., 1986, Brasília. Anais... Brasília: EMBRAPA-DDT/CNPq, 1986. p.65-69.

MARCHAL, J.; MALLESSARD, R. Comparison des immobilisations minerales de quatre cultivars de bananiers a fruits pour cuisson et de deux 'Cavendish'. Fruits, Paris, v.34, p.373392. 1979.

QUAGGIO, J.A.; RAIJ, B. van. Produtividade esperada. In: RAIJ, B. van; CANTARELLA, H.; QUAGGIO, J.A.; FURLANI, A.M.C. (Eds.) Recomendações de adubação e calagem para o Estado de São Paulo. 2.ed.rev.atual. Campinas: IAC, 1997.p.13-14. (Boletim Técnico, 100)
ROBINSON, J.C. Bananas and plantains. Wallingford: CAB International, 1996.238p.

SOTO, M. Bananos: cultivo y comercialización. $2^{\text {nd }}$ ed. San José: LIL, 1992. 674p.

TEIXEIRA, L.A.J.; RUGGIERO, C.; NATALE, W. Manutenção de folhas ativas em bananeira-'Nanicão' por meio do manejo das adubações nitrogenada e potássica e da irrigação. Revista Brasileira de Fruticultura, Jaboticabal, v.23, p.699-703, 2001.

TEIXEIRA, L.A.J.; SPIRONELLO,A.; QUAGGIO, J.A.; FURLANI, P. Banana. In: RAIJ, B. van; CANTARELLA, H.; QUAGGIO, J.A.; FURLANI, A.M.C. (Eds.) Recomendações de adubação e calagem para o Estado de São Paulo. 2.ed.rev.atual. Campinas: IAC, 1997. p.131-132. (Boletim Técnico, 100)

TURNER, D.W.; BARKUS, B. Yield, chemical composition, growth and maturity of 'Williams' banana fruit in relation to supply of potassium, magnesium and manganese. Scientia Horticulturae, Amsterdan, v.16, p.239-252, 1982. 